\title{
Ablation and Regeneration of Peripheral and Central TRPV1 Expressing Nerve Terminals and the Consequence of Nociception
}

\author{
Shuang-Quan $\mathrm{Yu}^{1,2, *}$ and Louis S. Premkumar ${ }^{1, *}$ \\ ${ }^{1}$ Department of Pharmacology, Southern Illinois University School of Medicine, Springfield, IL 62702, USA; \\ ${ }^{2}$ Department of Clinical Medicine, Michigan State University, Lansing, MI 48824, USA
}

\begin{abstract}
Transient Receptor Potential Vanilloid 1 (TRPV1) expressed in peripheral terminals is responsible for transducing thermal and chemical nociception. Role of TRPV1 expressed in the central terminals is not clear, however, its activation modulates synaptic transmission and contributes to central sensitization. In this study, we have determined the role of TRPV1 expressed in the peripheral and central terminals using resiniferatoxin (RTX), a potent TRPV1 agonist. A single intraplantar injection of RTX, within two days induced loss of capsaicin-induced nocifensive behavior and enhanced response latency to hot plate, which recovered over a period of two months. RTX treatment resulted in the ablation of peripheral TRPV1 expressing fibers in paw skin, which regenerated over the same time period. On the other hand, a single dose of intrathecal administration of RTX, within two days caused thermal hypoalgesia. RTX treatment ablated TRPV1 expressing central sensory nerve terminals. Intriguingly, in contrast to peripheral nerve terminal regeneration that occurred within two months, the central TRPV1 expressing nerve terminals did not regenerate even after five months. The present study demonstrates that TRPV1 in the peripheral and central terminals play a role in nociception and the peripheral terminals have the ability to regenerate, whereas the central terminals do not regenerate even after five months.
\end{abstract}

Keywords: Ablation, pain control, regeneration, resiniferatoxin, TRPV1.

\section{INTRODUCTION}

Transient Receptor Potential Vanilloid 1 (TRPV1) is a $\mathrm{Ca}^{2+}$ permeable non-specific cation channel. TRPV1 is predominately expressed in small diameter peptidergic dorsal root ganglion (DRG) and trigeminal ganglion neurons. TRPV1 is expressed in both peripheral and central terminals of the sensory neurons [1-5]. TRPV1 expressed at the peripheral terminals can be activated by a temperature ( $>43$ ${ }^{\circ} \mathrm{C}$ ) and as a result transduces thermal pain sensitivity to the brain via the spinal cord $[1,6]$. However, the role of TRPV1 expressed in the central terminals is far from clear. Activation of TRPV1 modulates synaptic transmission at the first sensory synapse between central terminals of DRG and dorsal horn neurons [1,5,7-11]. It is likely that peripheral injury-induced neuronal activity releases Calcitonin GeneRelated Peptide (CGRP) and Substance P (SP) from presynaptic nerve terminals and activation of glia influencing the synthesis and release of anandamide and proand anti-inflammatory mediators. Anandamide and inflammatory mediators act retrogradely to modulate synaptic transmission and TRPV1 expression and function, respectively [12-22]. Resiniferatoxin (RTX) is an ultrapotent TRPV1 agonist, which activates TRPV1 in an irreversible manner. As a result, TRPV1 nerve terminals are ablated by persistent increase in $\mathrm{Ca}^{2+}$ influx [5, 23-25]. Therefore, it would be useful to use RTX to target TRPV1 to

*Address correspondence to these authors at the Department of Clinical Medicine, Michigan State University, Lansing, MI 48824, USA; Tel: 217-545-2179; Fax: 217-545-0145;

E-mails: 1premkumar@siumed.edu and sqyu@msu.edu treat conditions such as peripherally mediated inflammatory pain including shingles, urinary bladder hyperreflexia, deiabetic peripheral neuropathy, arthritis etc. On the other hand, TRPV1 expressed at the central nerve terminals can be targeted to treat debilitating pain conditions such as pain associated with cancer. To test the effectiveness of intrathecal administration of RTX in treating cancer pain, a clinical trial has been initiated by National Institute of Dental and Craniofacial Research (NCT00804154). Usefulness of RTX has been shown in several modalities of pain [24, 26-31]. Karai et al. [26] reported that intraplantar administration of RTX produced thermal antinociception without affecting basal pain sensitivity. Neubert et al., [27] reported that regeneration of peripheral nerve endings was observed after RTX treatment. However more direct morphological and functional studies need to be conducted.

In order to study the role of TRPV1 expressed in peripheral and central terminals, we targeted TRPV1 by its antagonists or by RTX, which caused TRPV1 expressing nerve terminal ablation. We determined the nocifensive behavior to capsaicin, thermal sensitivity and the ability of the peripheral and central terminals to regenerate following RTX treatment.

\section{EXPERIMENTAL PROCEDURES}

\section{Animals}

TRPV1 knockout mice (B6.129S4-Trpv1tm1Jul/J) were purchased from The Jackson Laboratory and maintained as homozygous mutants [32]. Rats (SD, male, 250-300 g) and wild-type mice (C57B16/J, background strain of TRPV1 
knock-out, male, 25-30 g) were purchased from Harlan (Indianapolis, IN, USA). All animals were housed on a 12hour light/dark cycle with free access to food and water. Mice and rats were accustomed to the testing environment for 5 days prior to the first testing day to decrease the effect of stress. All experiments reported in this study were conducted in accordance with the protocols approved by the Laboratory Animal Care and Use Committee of Southern Illinois University School of Medicine.

\section{Ablation of TRPV1 Expressing Peripheral and Central Terminals}

$20 \mu \mathrm{l}$ of 1,3 or $10 \mu \mathrm{M}$ of RTX was injected intraplantarly into the left hind paw of mice to ablate the TRPV1 expressing peripheral terminals. $10 \mu \mathrm{l}$ of $100 \mu \mathrm{M}$ of RTX were injected intrathecally in rats to ablate the TRPV1 expressing central terminals.

\section{Nocifensive Behavior Test}

Capsaicin-evoked nocifensive behavior in mice was defined as licks/shakes [33]. The number of times the mouse licked/shook its left hind paw was counted over 3 min immediately after intraplantar administration of capsaicin $(100 \mu \mathrm{M}, 20 \mu \mathrm{l})$ or saline into the left hind paw.

\section{Hot Plate Test}

The hot plate test was used to measure response latencies to thermal stimulation. The mouse was placed on the heated surface of the hot plate apparatus (Columbus Instruments, Columbus, Ohio), which was maintained at $49.5 \pm 0.5^{\circ} \mathrm{C}$. The pain response was evaluated by recording the latency (s) for the mouse to shake or lick the hind paw or to jump from the hot plate (whichever occurred first). A cut-off time of $40 \mathrm{~s}$ was set to avoid injury to the paws. All mice were accustomed to the pain test conditions for 5 days before the experiments. The hot plate test was conducted before and repeated at 2, 4, 7, 14, 21, 28, 35, 42, 49, 56, 63 days after intraplantar injection of RTX or saline.

\section{Intrathecal Catheter Implantation and Intrathecal Injection}

Surgery: Intrathecal catheters were implanted in rats according to Yaksh and Rudy 1976, [34] with some modifications. Briefly, rats were anesthetized with ketamine/xylazine $(85 / 5 \mathrm{mg} / \mathrm{kg}$, i.p.). When they no longer responded to the tail pinch test, the nape area was shaved. The skin was swabbed with betadine followed by $70 \%$ alcohol. A small incision was made in the skin and the muscles were separated to expose the atlanto-occipital membrane (the dura mater between the atlas and os occipitale). A small incision was made in the membrane to allow a polyethylene- 10 catheter filled with $0.9 \%$ sterile saline to be inserted into the subarachnoid space. The catheter was threaded through the space as far as the lumbar enlargement $(7.5 \mathrm{~cm}$ from the atlanto-occipital membrane to the lumbar enlargement). The catheter was then sutured in place with the muscles and the incision closed. About $5 \mathrm{~cm}$ of catheter was left externally to act as an external port for injections. The external port was sealed with Parafilm film to ensure that the cerebrospinal fluid will not flow out via the catheter. Rats were allowed to recover for 7 days after surgery. To prevent infection, $10 \mathrm{mg} / \mathrm{kg}$ of kanamycin was injected subcutaneously everyday for 5 days during recovery. Intrathecal administration of drugs: Drugs $(10 \mu \mathrm{l})$ were administrated into the subarachnoid space via the catheter, which was flushed with $10 \mu$ of saline to ensure complete release of the drugs in the subarachnoid space.

\section{Radiant Heat Test}

To assess thermal nociceptive responses, a radiant heat instrument (Ugo Basile, Cat. No 7370) was employed [35]. The thermal nociceptive stimulus (radiant heat), originated from a focused projection bulb, was delivered to the plantar surface of rat hind paw. A timer was automatically activated with the light source, and paw withdrawal latency (PWL) was defined as the time required for the paw to show an abrupt withdrawal. Intensity of the heat source was adjusted to yield PWL ranging from 7 to $10 \mathrm{~s}$ for normal rats, and a cutoff time of $20 \mathrm{~s}$ was imposed to avoid tissue damage.

\section{Immunohistochemistry}

Mice or rats were anesthetized by ketamine/xylazine $(85 / 5 \mathrm{mg} / \mathrm{kg}$, ip). Freshly made fixative (4 \% paraformaldehyde) was perfused transcardially. L5 DRGs, the ventral skin of hind paw, and lumbar spinal cord were removed and kept in the same fixative for 3 hours, then incubated with $15 \%$ sucrose and $30 \%$ sucrose for 12 hours, respectively. Samples were quickly frozen with liquid nitrogen. DRG, skin and spinal cord were cut into sections with a thickness of 10,20 and $20 \mu \mathrm{m}$, respectively. After being perforated with PBS containing $0.1 \%$ Triton X-100 for 20 minutes and blocked with $10 \%$ donkey serum for 30 minutes, the sections of mouse samples were incubated with polyclonal rabbit anti-TRPV1 antibody (Calbiochem, PC420, 1:50) for one hour at room temperature, the sections of rat samples were incubated with polyclonal rabbit antiTRPV1 antibody (Affinity BioReagents, PA1-747, 1:500) and monoclonal mouse anti-NeuN antibody (Chemicon, MAB377, 1:100) for 1 hour at room temperature, then the sections were incubated with Rhodamine RedTM-X donkey anti-rabbit IgG (Jackson, 711-295-152, 1: 100) and FITC donkey anti-mouse IgG (Jackson, 715-095-151, 1: 100) for 1 hour at room temperature, then spread on slides, mounted with mounting medium (Biomeda Gel/Mount) and covered by cover slips. Pictures were taken by using confocal microscope (Olympus Fluoview).

The quantification of staining was as follows. The DRG was horizontally cut into sections. Only the full size sections (which include three intact parts, the two soma-concentrating lateral parts and the fiber-concentrating central part) were taken into account. The paw skin was longitudinally cut into sections. Only the TRPV1 staining in the small region between the second foot pad and the fifth foot pad was taken into account. The spinal cord was transversely cut into sections. Only the TRPV1 staining in the superficial layers of the dorsal horn was taken into account. Ten sections were selected randomly from these full size sections. The numbers of the stained TRPV1 neurons he total gray values were measured by ImageJ ((http://rsb.info.nih.gov/ij/). 


\section{Reagents}

RTX (R8756, Sigma-Aldrich) was dissolved in $95 \%$ ethanol and used as a stock solution. 1, 3 and $10 \mu \mathrm{M}$ of working concentrations of RTX were made by diluting the stock RTX in sterilized $0.9 \% \mathrm{NaCl}$. Capsaicin (M2028, Sigma-Aldrich) was dissolved in $95 \%$ ethanol and used as a stock solution. $100 \mu \mathrm{M}$ of working concentration of capsaicin was made by diluting the stock capsaicin in sterilized $0.9 \% \mathrm{NaCl}$. Capsazepine (C191, Sigma-Aldrich) was dissolved in $95 \%$ ethanol and used as a stock solution. $0.3,1$ and $3 \mathrm{mM}$ of working concentrations of capsazepine were made by diluting the stock capsaicin in sterilized $0.9 \%$ $\mathrm{NaCl}$.

\section{Statistical Analysis}

All data are reported as mean $\pm \mathrm{SE}$, differences between groups were analyzed statistically by the Student t-test with the level of significance set at $* \mathrm{P}<0.05$.

\section{RESULTS}

\section{Intraplantar Administration of RTX Abolished Capsaicin-induced Nocifensive Behavior in Mice}

RTX is a potent TRPV1 agonist that can activate the receptor irreversibly by binding with high affinity. In order to study the effect of RTX in the peripheral nerve terminals, a series of concentrations of $\operatorname{RTX}(1,3$ and $10 \mu \mathrm{M}, 20 \mu \mathrm{l})$ was injected into the plantar surface of mice $(n=4)$ left hind paw. Since capsaicin is a TRPV1 agonist that can induce nocifensive behavior when injected into the plantar region, we determined capsaicin $(100 \mu \mathrm{M}, 20 \mu \mathrm{l})$-induced nocifensive behavior in the RTX-treated mice every other day during the first week and once a week thereafter. A dose-dependent loss of capsaicin-induced nocifensive behavior was observed two days after RTX administration as compared to the control group. Especially higher concentration of RTX $(10 \mu \mathrm{M})$ caused complete loss of capsaicin-induced nocifensive behavior. Capsaicin-induced loss of nocifensive behavior gradually recovered to the basal level over a period of sixty three days following treatment with RTX (Fig. 1).

\section{Intraplantar Injection of RTX Enhanced the Response Latency to Hot Plate in Mice}

Once it was determined that $10 \mu \mathrm{M}$ of RTX was an effective concentration, we next examined the changes in thermal sensation in mice $(n=13-25)$ treated with $10 \mu \mathrm{M}$ intraplantar RTX. A strong thermal anti-nociception was observed in the RTX-treated mice indicated by the enhanced response latency to the hot plate test, which remarkably increased to the cut-off time (40 s) within two days following RTX treatment. The mice were subjected to hot plate test twice a week for the first three weeks and once a week thereafter. The response latency decreased gradually over a period of 2 months. The control mice injected with saline showed no change in response latency, when subjected to hot plate test. We also used TRPV1 knockout mice that showed a moderate increase in response latency as compared to RTX-treated animals (Fig. 2).

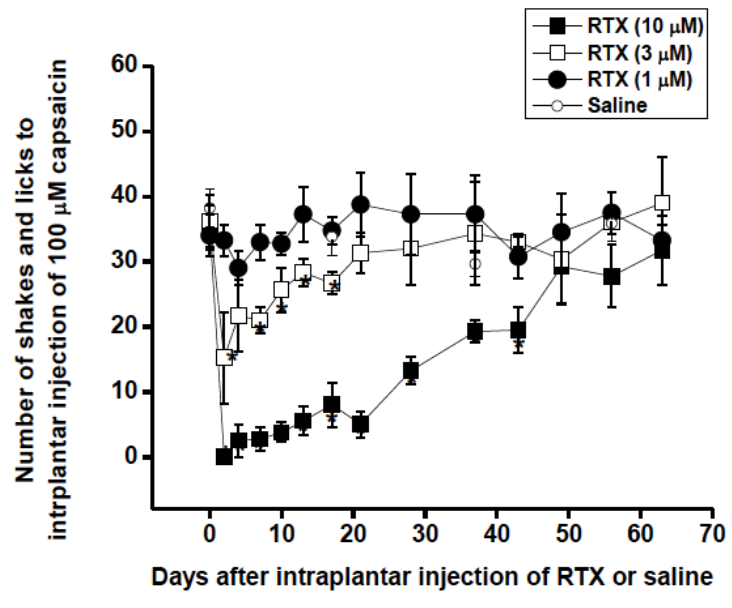

Fig. (1). Effects of intraplantar administration of RTX on capsaicininduced nocifensive behavior in mice. Time $=0$ day: intraplantar injection of $20 \mu \mathrm{l}$ of 1,3 or $10 \mu \mathrm{M}$ of RTX, or $20 \mu \mathrm{l}$ of $0.9 \%$ saline as the control group. Data are presented as mean $\pm \mathrm{SE}$, number of shakes and licks to intraplantar injection of capsaicin $(100 \mu \mathrm{M}, 20$ $\mu \mathrm{l})$. Horizontal axis indicate days after the RTX injection. The statistical difference at time points between groups was evaluated by Student's t-test. * $\mathrm{P}<0.05$ compared with the control group, 20 $\mu 1$ of $0.9 \%$ saline.

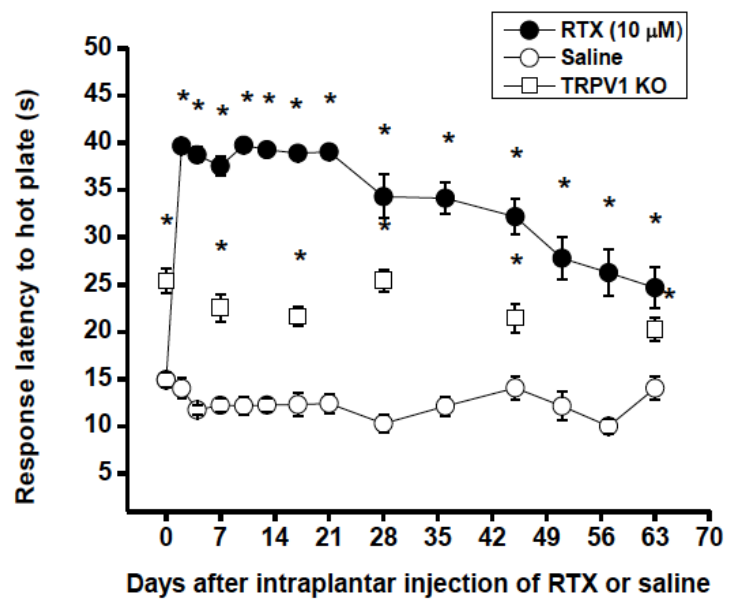

Fig. (2). Effects of intraplantar administration of RTX on response latency to hot plate in mice. Time $=0$ day: intraplantar injection of $20 \mu \mathrm{l}$ of $10 \mu \mathrm{M}$ of RTX, or $20 \mu \mathrm{l}$ of $0.9 \%$ saline as the control group. Another control group was TRPV1 knockout mice. Data are presented as mean $\pm \mathrm{SE}$, nocifensive behavior latency to hot plate (s). Horizontal axis indicate days after the RTX injection. The statistical difference at time points between groups was evaluated by Student's t-test. * $\mathrm{P}<0.05$ compared with the control group, 20 $\mu 1$ of $0.9 \%$ saline.

\section{Ablation and Regeneration of Peripheral TRPV1 Expressing Nerve Terminals by Intraplantar Administration of RTX}

In order to determine whether the loss and recovery of capsaicin-induced nocifensive behavior and the thermal antinociception were due to ablation and regeneration of TRPV1 expressing nerve fibers, we used immunohistochemical methods and stained for TRPV1 expressing fibers. The paw skin of the RTX- and salinetreated mice were collected at different days following 
treatment. There was no TRPV1-staining observed in mouse paw skin, within two days of intraplantar administation of RTX $(10 \mu \mathrm{M}, 20 \mu \mathrm{l})$. However, the nerve fibers regenerated gradually over a period of time and a substantial regeneration of TRPV1-expressing fibers was observed in mouse paw skin over the period of two months (Fig. 3). We also examined the change in TRPV1 expressing cell bodies by immunostaining the L5 DRG. There was neither a change in the number of neuronal cell bodies stained nor in the intensity of staining (Fig. 4).

\section{Intrathecal Administration of TRPV1 Antagonist Capsazepine Induced Anti-nociception in Rats}

Then, we examined the role of TRPV1 expressed in central terminals by administering capsazepine $(0.3,1$ and 3 $\mathrm{mM}, 10 \mu \mathrm{l})$ into the subarachnoid space of rats $(\mathrm{n}=8)$. A short-term transient dose-dependent thermal hypoalgesia was observed after capsazepine administration as compared to the control group (Fig. 5). This suggests that block of TRPV1 expressed in the central terminals plays a role in thermal nociception. The transient nature of response might be due to diffusion of the antagonist in the cerebrospinal fluid.

\section{Intrathecal Administration of RTX Enhanced the PWL to Radiant Heat in Rats}

Since peripheral TRPV1 expressing nerve terminal ablation by RTX resulted in thermal hypoalgesia, which recovered over a period of 2 months, we wanted to determine the role of TRPV1 expressed in the central nerve terminals by intrathecal administration of RTX $(100 \mu \mathrm{M}, 10 \mu \mathrm{l})$. We chose rats for these studies because of the ease with which intrathecal administration can be performed. However, We will have to take into account effects associated with species difference. PWL was determined using a radiant heat source and testing was carried out every four days over a period of three weeks. There was a significant increase in PWL following administation of a single dose of RTX (Fig. 6). Most interesting observation is that unlike following intraplantar RTX administration (Figs. 1 and 2), the PWL did not decrease or recover over time $(n=5-6)$. We have attributed the recovery of thermal sensitivity following peripheral RTX administration to regeneration of the nerve terminals.

In order to exclude the possibility of nonspecific effects, we conducted the experiments by intrathecal administration

A
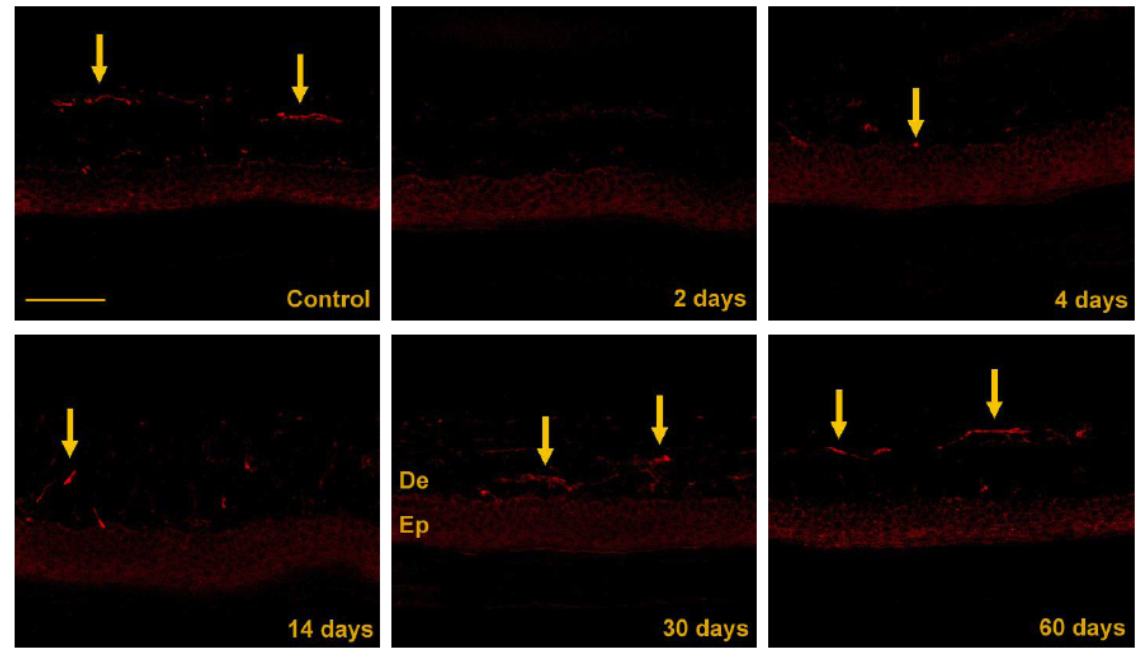

B

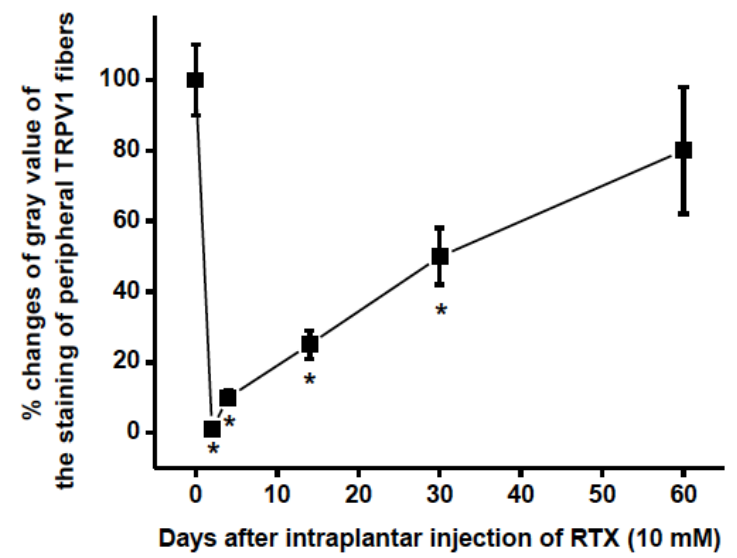

Fig. (3). TRPV1 staining in paw skin of mice at different day after RTX treatment (A). Arrows show the TRPV1 fibers in the epidermis (Ep) and dermis (De). Scale bar, $50 \mu \mathrm{m}$. The quantification of the gray value of the TRPV1 staining is shown in graph $\mathbf{B}$. 
A

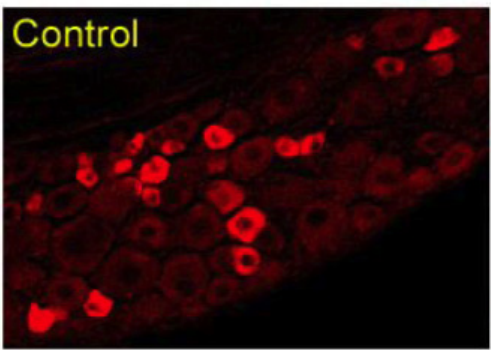

B

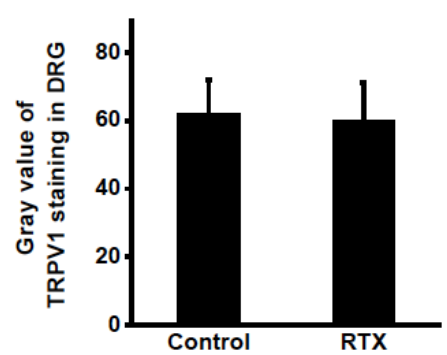

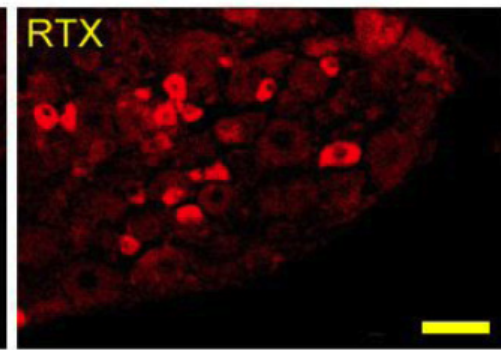

C

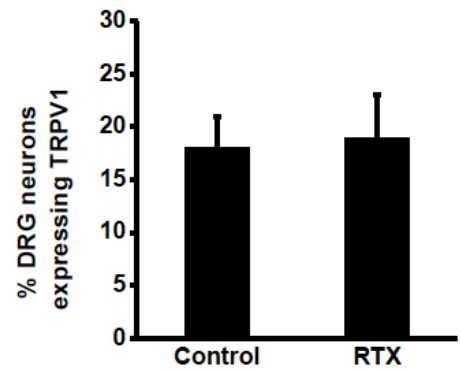

Fig. (4). TRPV1 staining in DRG from RTX treatment mice and the control mice (A). Scale bar, $25 \mu \mathrm{m}$. The quantification of the gray value of TRPV1 staining in DRG and the percentage of TRPV1-expressing DRG neurons are shown in graph $\mathbf{B}$ and $\mathbf{C}$, respectively.

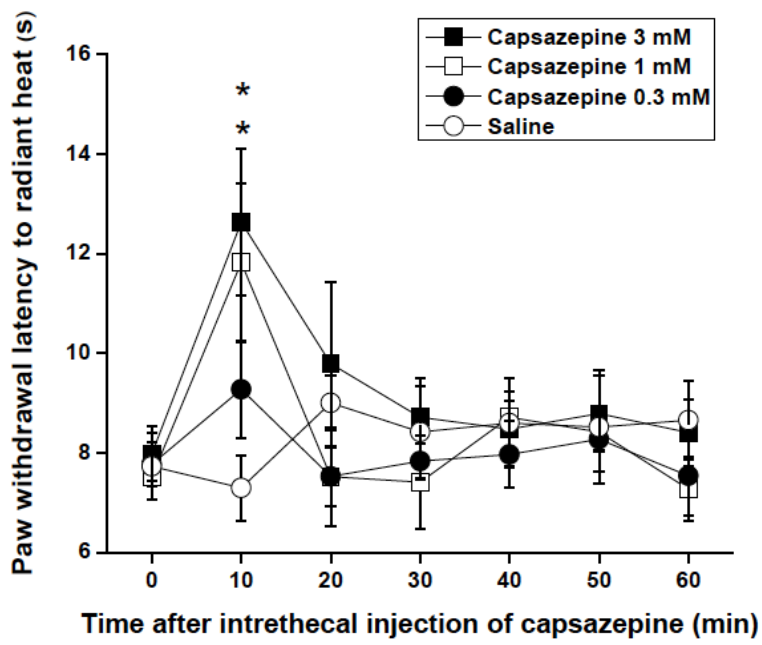

Fig. (5). Effects of intrathecal administration of capsazepine on PWL (paw withdrawal latency) to radiant heat in rats. Time $=0 \mathrm{~min}$ : intrathecal injection of $10 \mu 1$ of $0.3,1$ and $3 \mathrm{mM}$ of capsazepine, or $10 \mu \mathrm{l}$ of $0.9 \%$ saline as the control group. Data are presented as mean $\pm \mathrm{SE}$, paw withdrawal latency to radiant heat (s). Horizontal axis indicate time $(\mathrm{min})$ after the capsazepine injection. The statistical difference at time points between groups was evaluated by Student's t-test. * $\mathrm{P}<0.05$ compared with the control group.

of capsazepine in RTX-treated rats $(10 \mu \mathrm{M}, 10 \mu 1$ at 7 days after RTX) ( $\mathrm{n}=8)$, in which the TRPV1 expressing central nerve terminals were ablated. There was no change in thermal pain sensitivity suggesting that the common target for capsazepine and RTX is TRPV1 (Fig. 7).

\section{Ablation and Regeneration of TRPV1 Expressing Central Sensory Nerve Terminals by RTX}

In order to further examine the effects of intrathecal RTX administration, we used immunohistochemistry. The spinal

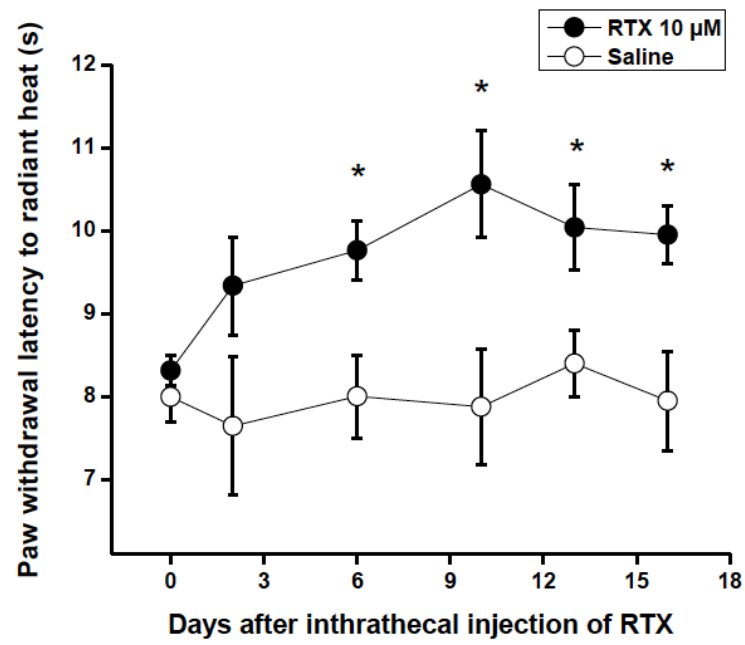

Fig. (6). Time-course changes of PWL to radiant heat in rats received treatment of intrathecal administration of RTX. Time $=0$ day: intrathecal injection of $10 \mu \mathrm{l}$ of $100 \mu \mathrm{M}$ of RTX, or $10 \mu \mathrm{l}$ of $0.9 \%$ saline as the control group. Data are presented as mean $\pm \mathrm{SE}$, paw withdrawal latency to radiant heat (s). Horizontal axis indicate days after the RTX injection. The statistical difference at time points between groups was evaluated by Student's t-test. * $\mathrm{P}<0.05$ compared with the control group.

cord tissue was collected after seven days following intrathecal administration of RTX $(100 \mu \mathrm{M}, 10 \mu \mathrm{l})$ and stained for TRPV1. There was no TRPV1 labeling observed in dorsal horn following RTX treatment, however labeling of dorsal horn neuronal cell bodies with NeuN, a neuronal marker was unaffected.

Since there was no recovery from enhanced PWL, we determined the regeneration of central terminals over a period by staining the spinal cord tissue for TRPV1 at different time points. Intriguingly, even after 5 months 
following RTX treatment, there was no TRPV1 staining observed in the dorsal horn suggesting that TRPV1 expressing central terminals did not regenerate after RTX administration (Fig. 8).

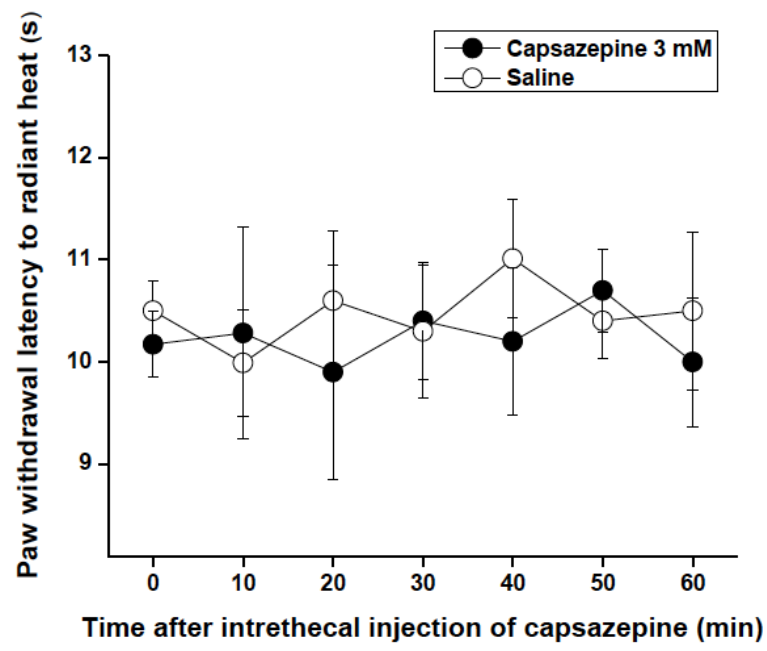

Fig. (7). Effects of intrathecal administration of capsazepine on PWL to radiant heat in central TRPV1-ablated rats by RTX. Time $=$ $0 \mathrm{~min}$ : intrathecal injection of $10 \mu \mathrm{l}$ of $3 \mathrm{mM}$ of capsazepine, or 10 $\mu 1$ of $0.9 \%$ saline as the control group. Data are presented as mean $\pm \mathrm{SE}$, paw withdrawal latency to radiant heat (s). Horizontal axis indicates time ( $\mathrm{min}$ ) after the capsazepine. The statistical difference at time points between groups was evaluated by Student's t-test. $* \mathrm{P}<0.05$ compared with the control group.

It could be argued that the DRG neuronal cell bodies were ablated by intrathecal administration of RTX, hence the inability of the central terminals to regenerate. Therefore, we determined the number of TRPV1 expressing neurons and their intensity of staining in L5 DRG in RTX-treated animals. There was no difference in the number of TRPV1 expressing neurons or in their intensity of staining (Fig. 9). Based on this observation, it is tempting to suggest that central TRPV1 expressing sensory nerve terminals do not have the ability to regenerate as compared to peripheral sensory nerve terminals.

\section{DISCUSSION}

Ablation of peripheral sensory nerve terminals by a single intraplantar injection of RTX resulted in the loss of TRPV1 staining in plantar skin, which correlated with the loss of capsaicin-induced nocifensive behavior and thermal sensitivity. Over a period of two months, a gradual recovery of nocifensive behavior, thermal sensitivity and TRPV1 staining were observed, suggesting regeneration of ablated nerve terminals.

Ablation of central sensory nerve terminals by a single intrathecal administration of RTX resulted in the loss of TRPV1 staining in the laminae I and II of the spinal cord and enhanced PWL to radiant heat. However, unlike the peripheral nerve terminals, neither the PWL nor the TRPV1 staining recovered, suggesting the inability of the central terminals to regenerate.

As alluded to earlier, it could be argued that the DRG neuronal loss by the intrathecal administration of RTX could prevent the regeneration, as it has been shown that intrathecal delivery of antisense oligodeoxynucleotide can be retrogradely transported in sufficient concentrations that can induce DRG neuronal cell death [36]. In order to test this possibility, we stained the L5 DRG for TRPV1. Following intrathecal administration of RTX, the number of cell bodies stained for TRPV1 remained the same and the intensity of staining was also unaffected as compared to control saline administered animals.

A

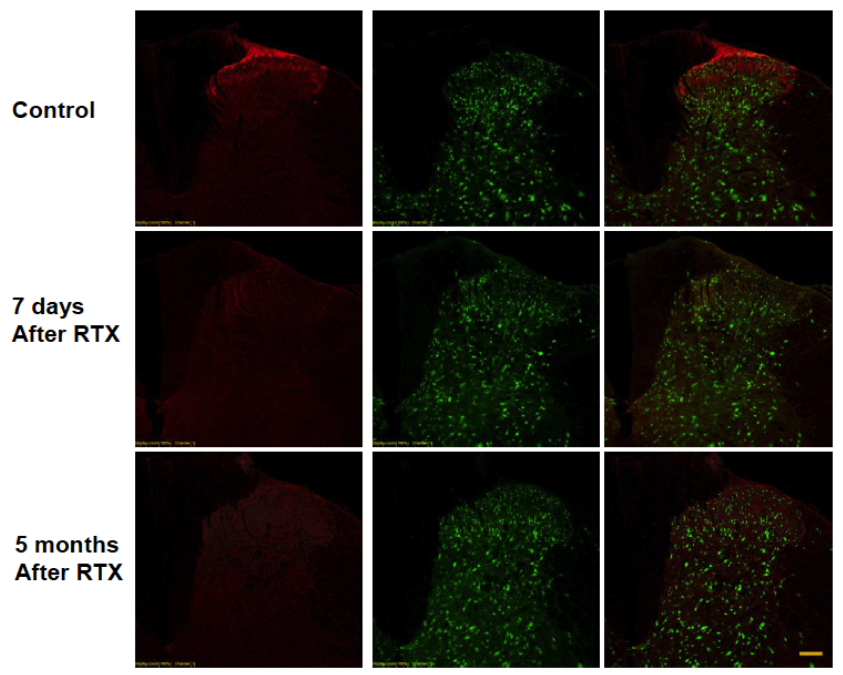

B

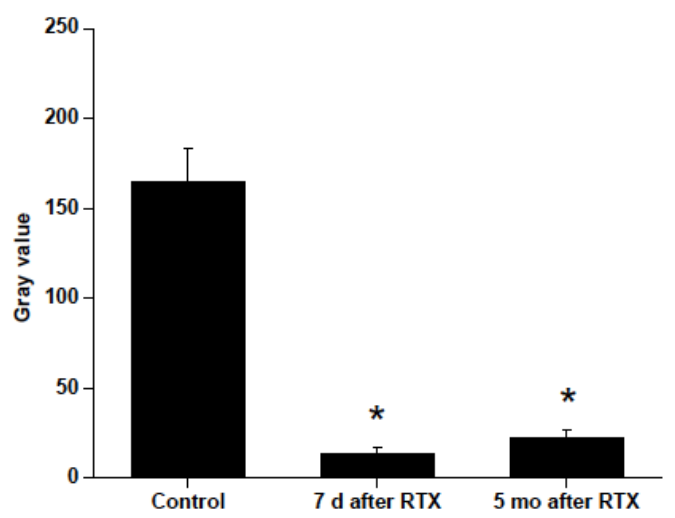

Fig. (8). Intrathecal administration of RTX ablate TRPV1 expressing central sensory nerve terminals in rats. Staining of TRPV1 and NeuN (a neuron cell body marker) in the dorsal horn of the L5 lumbar segment of the spinal cord from control rats and RTX-treated rats (A). As compared with control rats, TRPV1 staining in RTX-treated rats was not observed in the dorsal horn of L5 spinal cord. RTX treatment didn't affect the dorsal horn neurons, as shown by NeuN staining. The central TRPV1 expressing central terminals are incapable of regenerating after RTX-induced ablation. Scale bar, $50 \mu \mathrm{m}$. The quantification of the gray value of the TRPV1 staining is shown in graph $\mathbf{B}$.

TRPV1 is a polymodal receptor involved in the transduction of chemical and thermal sensitivities. Interestingly, the antinociceptive effect of RTX-treated mice was greater than TRPV1 knockout mice, which may suggest other nociceptive receptors such as TRPV2, TRPV3 or TRPM8 may play a role in thermal nociception. Studies have 
A

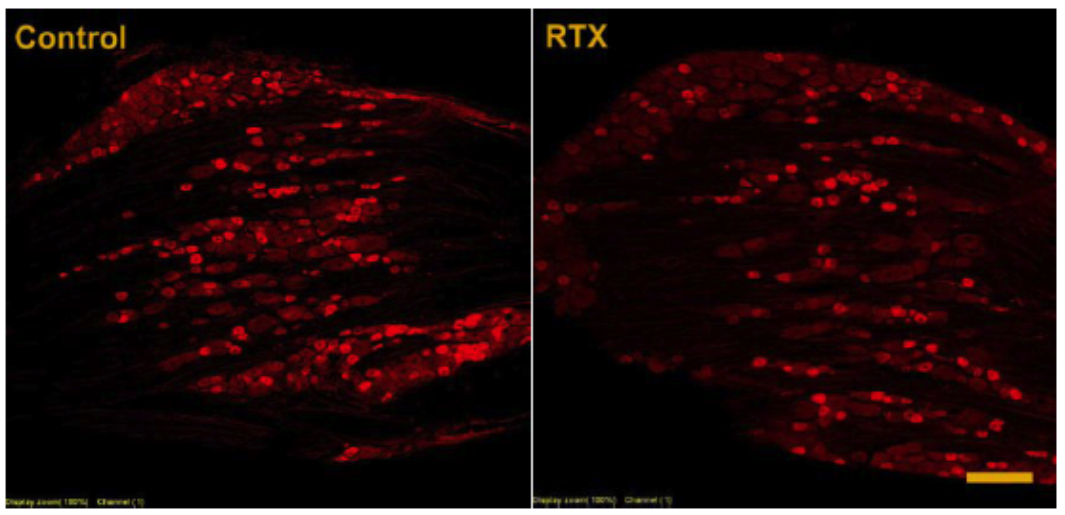

B

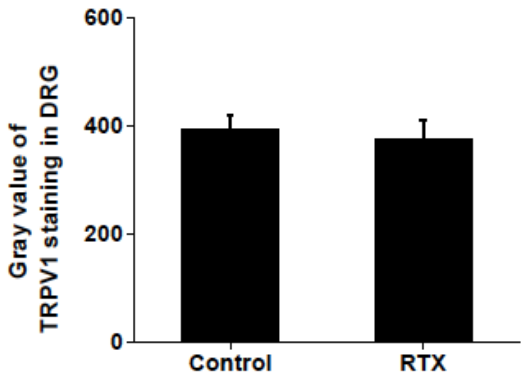

C

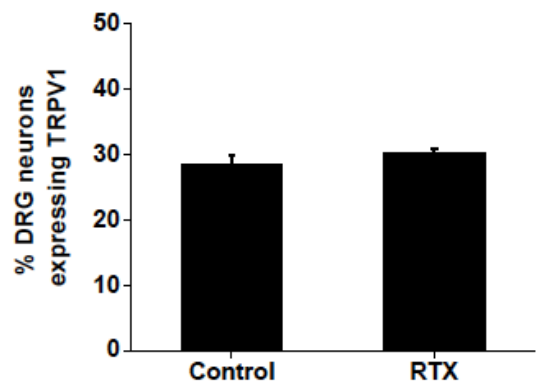

Fig. (9). Degeneration of TRPV1 expressing central nerve terminals after intrathecal RTX in rats did not affect the TRPV1 expressing DRG neuronal number or the intensity of their staining (A). Scale bar, $50 \mu \mathrm{m}$. The quantification of the gray value of TRPV1 staining in DRG and the percentage of TRPV1-expressing DRG neurons are shown in graph $\mathbf{B}$ and $\mathbf{C}$, respectively.

shown that TRPV1 and TRPV2, TRPV3, NK1, SP and CGRP are co-localized in the same DRG neuron [6, 37-40]. Ablation of TRPV1-expressing nerve terminals will also eliminate other nociceptive ion channels and metabotropic receptors that modulate TRPV1 function. It should also be taken into consideration, the role of TRPV1 expressed in the gabargic interneurons in the spinal cord [41].

There is a dense distribution of TRPV1 in dorsal horn of spinal cord of rats and mice [1,6,5], which suggests that there may be functions associated with central TRPV1, although yet to be demonstrated. In this study, we have demonstrated that thermal anti-nociception could be induced by blocking TRPV1 by its antagonist, capsazepine. This was confirmed by the observation that thermal hypoalgesia occurred following intrathecal administration of ultra-potent TRPV1 agonist, RTX. Taken together these observations demonstrate that TRPV1 in the dorsal horn of rat spinal cord plays a role in thermal nociception. Besides, intrathecal administration of RTX ablated only TRPV1 expressing central terminals rendering a novel strategy to selectively study the role of TRPV1 in the central terminals of the sensory neurons.

Administration of intrathecal RTX resulted in the loss of TRPV1 staining, which is interpreted as ablation of TRPV1 expressing nerve terminals. The loss of thermal sensation and TRPV1 staining in the spinal dorsal horn following intrathecal administration of RTX did not recover over a period of five months, suggesting the inability of the central nerve terminals to regenerate following RTX treatment. One of the criticisms for the strategy of intrathecal administration of RTX is that, although the pain relief is effective, the process of regeneration could make the pain condition worse because of indiscriminate, unorganized and excessive nerve fiber regeneration. However, it was surprising to learn that the central terminal did not regenerate following RTXinduced ablation. Therefore, the danger of excessive regeneration can be prevented; nevertheless, ablating central nerve terminals is not a preferred outcome that might have long-term consequences. It is commendable that a clinical trial has begun specifically to treat terminal debilitating cancer pain conditions (National Institute of Dental and Craniofacial Research, NCT00804154).

Injury-induced neuronal activity releases CGRP and SP from the central nerve endings of the sensory neurons, which is partly mediated by TRPV1. Once released, these neuropeptides bind to their receptors on neurons and glia and trigger downstream signaling events leading to central sensitization [12-22]. Neuronal activity increases endovanilloid levels during chronic pain conditions, which are synthesized and released on demand [42-46]. Studies have shown that blocking neuropeptide release alleviates inflammatory [47-49] and neuropathic pain [50]. Drugs that inhibit activation of glia have been shown to alleviate chronic pain [51]. Similarly, gene therapy that increases the levels of anti-inflammatory mediators has been shown to alleviate chronic pain [52-54]. TRPV1 expressed in the central terminals could be a target for modulation by these processes.

\section{CONCLUSION}

In conclusion, our studies show that RTX administration can ablate peripheral TRPV1 expressing nerve fibers 
temporally without affecting their regeneration capability. The accompanying effects of loss of nocifensive behavior and thermal hypoalgesia recovered overtime shed insight into potential therapeutic strategies that can be developed to treat certain modalities of pain involving peripheral nerves such as diabetic peripheral neuropathy, shingles, urinary bladder hyperreflexia and arthritis. On the other hand, intrathecal administation of TRPV1 agonists can provide long-lasting pain relief by ablating TRPV1 expressing central sensory nerve terminals in the dorsal horn. Although RTX is in clinical trials, nerve terminal ablation is not a preferred outcome. However, further research can be undertaken to cause partial ablation by manipulating concentrations of RTX or using less potent TRPV1 agonists.

\section{CONFLICT OF INTEREST}

The authors confirm that this article content has no conflict of interest.

\section{ACKNOWLEDGEMENTS}

This work was supported with grants from NIH (DK065742, NS042296 to L.S.P) and SIU Excellence in Academic Medicine (L.S.P).

\section{PATIENT'S CONSENT}

Declared none.

\section{REFERENCES}

[1] Caterina MJ, Schumacher MA, Tominaga M, et al. The capsaicin receptor: a heat-activated ion channel in the pain pathway. Nature 1997; 389: 816-24.

[2] Dinh QT, Groneberg DA, Peiser C, et al. Substance P expression in TRPV1 and trkA-positive dorsal root ganglion neurons innervating the mouse lung. Respir Physiol Neurobiol 2004; 144: 15-24.

[3] Lazzeri M, Vannucchi MG, Zardo C, et al. Immunohistochemical evidence of vanilloid receptor 1 in normal human urinary bladder. Eur Urol 2004; 46: 792-8.

[4] Sikand P, Premkumar LS. Potentiation of glutamatergic synaptic transmission by protein kinase C-mediated sensitization of TRPV1 at the first sensory synapse. J Physiol 2007; 581: 631-47.

[5] Jeffry JA, Yu SQ, Sikand P, et al. Selective targeting of TRPV1 expressing sensory nerve terminals in the spinal cord for long lasting analgesia. PLoS One 2009; 4: e7021.

[6] Tominaga M, Caterina MJ, Malmberg AB, et al. The cloned capsaicin receptor integrates multiple pain-producing stimuli. Neuron 1998; 21: 531-43.

[7] Tognetto M, Amadesi S, Harrison S, et al. Anandamide excites central terminals of dorsal root ganglion neurons via vanilloid receptor-1 activation. J Neurosci 2001; 21: 1104-9.

[8] Nakatsuka T, Furue H, Yoshimura M, et al. Activation of central terminal vanilloid receptor-1 receptors and alpha beta-methyleneATP-sensitive $\mathrm{P} 2 \mathrm{X}$ receptors reveals a converged synaptic activity onto the deep dorsal horn neurons of the spinal cord. J Neurosci 2002; 22: 1228-37.

[9] Baccei ML, Bardoni R, Fitzgerald M. Development of nociceptive synaptic inputs to the neonatal rat dorsal horn: glutamate release by capsaicin and menthol. J Physiol 3003; 549: 231-42.

[10] Wang H, Kohno T, Amaya F, et al. Bradykinin produces pain hypersensitivity by potentiating spinal cord glutamatergic synaptic transmission. J Neurosci 2005; 25: 7986-92.

[11] Horvath G, Kekesi G, Nagy E, et al. The role of TRPV1 receptors in the antinociceptive effect of anandamide at spinal level. Pain 2008; 134: 277-84.

[12] Wimalawansa SJ. Calcitonin gene-related peptide and its receptors: molecular genetics, physiology, pathophysiology, and therapeutic potentials. Endocr Rev 1996; 17: 533-85.
[13] van Rossum D, Hanisch UK, Quirion R. Neuroanatomical localization, pharmacological characterization and functions of CGRP, related peptides and their receptors. Neurosci Biobehav Rev 1997; 21: 649-78.

[14] Ji RR, Woolf CJ. Neuronal plasticity and signal transduction in nociceptive neurons: implications for the initiation and maintenance of pathological pain. Neurobiol Dis 2001; 8: 1-10.

[15] Hains BC, Waxman SG. Activated microglia contribute to the maintenance of chronic pain after spinal cord injury. J Neurosci 2006; 26: 4308-17.

[16] Scholz J, Woolf CJ. The neuropathic pain triad: neurons, immune cells and glia. Nat Neurosci 2007; 10: 1361-68.

[17] Sappington RM, Calkins DJ. Contribution of TRPV1 to microgliaderived IL-6 and NFkappaB translocation with elevated hydrostatic pressure. Invest Ophthalmol Vis Sci 2008; 49: 3004-17.

[18] Watkins LR, Milligan ED, Maier SF. Glial proinflammatory cytokines mediate exaggerated pain states: implications for clinical pain. Adv Exp Med Biol 2003; 521: 1-21.

[19] Aoki Y, Ohtori S, Takahashi K, et al. Expression and co-expression of VR1, CGRP, and IB4-binding glycoprotein in dorsal root ganglion neurons in rats: differences between the disc afferents and the cutaneous afferents. Spine 2005; 30: 1496-1500.

[20] Chattopadhyay S, Myers RR, Janes J, et al. Cytokine regulation of MMP-9 in peripheral glia: implications for pathological processes and pain in injured nerve. Brain Behav Immun 2007; 21: 561-68.

[21] Westlund KN, Kochukov MY, Lu Y, et al. Impact of central and peripheral TRPV1 and ROS levels on proinflammatory mediators and nociceptive behavior. Mol Pain 2010; 6: 46.

[22] Woolf CJ. Central sensitization: implications for the diagnosis and treatment of pain. Pain 2011; 152: S2-15.

[23] Bishnoi M, Bosgraaf CA, Abooj M, et al. Streptozotocin-induced early thermal hyperalgesia is independent of glycemic state of rats: role of transient receptor potential vanilloid 1(TRPV1) and inflammatory mediators. Mol Pain 2011; 7: 52.

[24] Brown DC, Iadarola MJ, Perkowski SZ, et al. Physiologic and antinociceptive effects of intrathecal resiniferatoxin in a canine bone cancer model. Anesthesiology 2005; 103: 1052-59.

[25] Raisinghani M, Pabbidi RM, Premkumar LS. Activation of transient receptor potential vanilloid 1 (TRPV1) by resiniferatoxin. J Physiol 2005; 567: 771-86.

[26] Karai L, Brown DC, Mannes AJ, et al. Deletion of vanilloid receptor 1-expressing primary afferent neurons for pain control. J Clin Invest 2004; 113: 1344-52.

[27] Neubert JK, Karai L, Jun JH, et al. Peripherally induced resiniferatoxin analgesia. Pain 2003; 104: 219-28.

[28] Lee MG, Huh BK, Choi SS, et al. The effect of epidural resiniferatoxin in the neuropathic pain rat model. Pain Physician 2012; 15: 287-96.

[29] Pan HL, Khan GM, Alloway KD, et al. Resiniferatoxin induces paradoxical changes in thermal and mechanical sensitivities in rats: mechanism of action. J Neurosci 2003; 23: 2911-19.

[30] Szabo $\mathrm{T}$, Olah Z, Iadarola MJ, et al. Epidural resiniferatoxin induced prolonged regional analgesia to pain. Brain Res 1999; 840: 92-8.

[31] Brown DC, Agnello K, Iadarola MJ. Intrathecal resiniferatoxin in a dog model: Efficacy in bone cancer pain. Pain. 2015 Feb 5. [Epub ahead of print].

[32] Caterina MJ, Leffler A, Malmberg AB, et al. Impaired nociception and pain sensation in mice lacking the capsaicin receptor. Science 2000; 288: 306-13.

[33] Gilchrist HD, Allard BL, Simone DA. Enhanced withdrawal responses to heat and mechanical stimuli following intraplantar injection of capsaicin in rats. Pain 1996; 67: 179-88.

[34] Yaksh TL, Rudy TA. Analgesia mediated by a direct spinal action of narcotics. Science 1976; 192: 1357-58.

[35] Hargreaves K, Dubner R, Brown F, et al. A new and sensitive method for measuring thermal nociception in cutaneous hyperalgesia. Pain 1988; 32: 77-88.

[36] Lai J, Gold MS, Kim CS, et al. Inhibition of neuropathic pain by decreased expression of the tetrodotoxin-resistant sodium channel, NaV1.8. Pain 2002; 95: 143-52.

[37] Guo A, Vulchanova L, Wang J, et al. Immunocytochemical localization of the vanilloid receptor 1 (VR1): relationship to neuropeptides, the P2X3 purinoceptor and IB4 binding sites. Eur J Neurosci 1999; 11: 946-58. 
[38] Ständer S, Moormann C, Schumacher M, et al. Expression of vanilloid receptor subtype 1 in cutaneous sensory nerve fibers, mast cells, and epithelial cells of appendage structures. Exp Dermatol 2004; 13: 129-39.

[39] $\mathrm{Xu} \mathrm{H}$, Ramsey IS, Kotecha SA, et al. TRPV3 is a calciumpermeable temperature-sensitive cation channel. Nature 2002; 418: 181-86.

[40] Tsukagoshi M, Goris RC. Funakoshi K. Differential distribution of vanilloid receptors in the primary sensory neurons projecting to the dorsal skin and muscles. Histochem Cell Biol 2006; 126: 343-52.

[41] Kim YH, Back SK, Davies AJ, et al. TRPV1 in GABAergic interneurons mediates neuropathic mechanical allodynia and disinhibition of the nociceptive circuitry in the spinal cord. Neuron 2012; 74: 640-7.

[42] Bradshaw HB, Walker JM. The expanding field of cannabimimetic and related lipid mediators. Br J Pharmacol 2005; 144: 459-65.

[43] Pacher P, Bátkai S, Kunos G. The endocannabinoid system as an emerging target of pharmacotherapy. Pharmacol Rev 2006; 58: $389-462$.

[44] Hill L, Schug SA. Recent advances in the pharmaceutical management of pain. Expert Rev Clin Pharmacol 2009; 2: 543-57.

[45] Piomelli D. The molecular logic of endocannabinoid signalling. Nat Rev Neurosci 2003; 4: 873-84.

[46] Di Marzo V, De Petrocellis L, Bisogno T. The biosynthesis, fate and pharmacological properties of endocannabinoids. Handb Exp Pharmacol 2005; 168: 147-85.
[47] Yu LC, Hansson P, Brodda-Jansen G, et al. Intrathecal CGRP8-37induced bilateral increase in hindpaw withdrawal latency in rats with unilateral inflammation. Br J Pharmacol 1996; 117: 43-50.

[48] Yu LC, Hansson P, Lundeberg S, et al. Effects of calcitonin generelated peptide-(8-37) on withdrawal responses in rats with inflammation. Eur J Pharmacol 1998; 347: 275-82.

[49] Sun RQ, Lawand NB, Willis WD. The role of calcitonin generelated peptide (CGRP) in the generation and maintenance of mechanical allodynia and hyperalgesia in rats after intradermal injection of capsaicin. Pain 2003; 104: 201-08.

[50] Bennett AD, Chastain KM, Hulsebosch CE. Alleviation of mechanical and thermal allodynia by $\operatorname{CGRP}(8-37)$ in a rodent model of chronic central pain. Pain 2000; 86: 163-75.

[51] Raghavendra V, Tanga F, DeLeo JA. Inhibition of microglial activation attenuates the development but not existing hypersensitivity in a rat model of neuropathy. J Pharmacol Exp Ther 2003; 306: 624-30.

[52] Woods JM, Katschke KJ Jr, Tokuhira M, et al. Reduction of inflammatory cytokines and prostaglandin E2 by IL-13 gene therapy in rheumatoid arthritis synovium. J Immunol 2000; 165: 2755-63.

[53] Hao S, Mata M, Glorioso JC, et al. HSV-mediated expression of interleukin-4 in dorsal root ganglion neurons reduces neuropathic pain. Mol Pain 2006; 2 : 6.

[54] Milligan ED, Langer SJ, Sloane EM, et al. Controlling pathological pain by adenovirally driven spinal production of the antiinflammatory cytokine, interleukin-10. Eur J Neurosci 2005; 21: 2136-48.

Received: September 23, 2014

Revised: February 25, 2015

Accepted: February 27, 2015

(C) Yu and Premkumar; Licensee Bentham Open.

This is an open access article licensed under the terms of the Creative Commons Attribution Non-Commercial License (http://creativecommons.org/licenses/by-nc/3.0/) which permits unrestricted, non-commercial use, distribution and reproduction in any medium, provided the work is properly cited. 